\title{
A new approach for locating the minor apical foramen using an artificial neural network
}

\author{
M. A. Saghiri ${ }^{1}$, K. Asgar ${ }^{2}$, K. K. Boukani ${ }^{3}$, M. Lotfi ${ }^{4}$, H. Aghili ${ }^{5}$, A. Delvarani ${ }^{6}$, K. Karamifar $^{6}$, \\ A. M. Saghiri ${ }^{7}$, P. Mehrvarzfar ${ }^{6}$ \& F. Garcia-Godoy ${ }^{8}$ \\ ${ }^{1}$ Department of Dental Material, Dental Branch, Islamic Azad University, Tehran, Iran; ${ }^{2}$ Department of Dental and Biological \\ Materials, University of Michigan, MI, USA; ${ }^{3}$ Private practice, Tehran, Iran; ${ }^{4}$ Research Center for Pharmaceutical Nanotechnology \\ and Department of Endodontics, Dental Faculty, Tabriz University (Medical Sciences), Tabriz, Iran; ${ }^{5}$ IBM Software Group, \\ Healthcare and Life Sciences, NY, USA; ${ }^{6}$ Department of Endodontics, Islamic Azad University, Tehran, Iran; ${ }^{7}$ Department of \\ Biomedical Engineering, Kamal Asgar Research Center (KARC), Tehran, Iran; and ${ }^{8}$ College of Dentistry, University of Tennessee, \\ Memphis, TN, USA
}

\begin{abstract}
Saghiri MA, Asgar K, Boukani KK, Lotfi M, Aghili H, Delvarani A, Karamifar K, Saghiri AM, Mehrvarzfar $P$, Garcia-Godoy F. A new approach for locating the minor apical foramen using an artificial neural network. International Endodontic Journal, 45, 257-265, 2012.
\end{abstract}

Aim To develop a new approach for locating the minor apical foramen (AF) using feature-extracting procedures from radiographs and then processing data using an artificial neural network (ANN) as a decisionmaking system.

Methodology Fifty straight single-rooted teeth were selected and placed in a socket within the alveolar bone of a dried skull. Access cavities were prepared and a file was place in the canals to determine the working length. A radiograph was taken to evaluate the location of the file in relation to the minor foramen and further checked after retrieving the tooth from the alveolar socket. The location of the file tip was categorized into: beyond the AF (long), within the root canal (short) and just at the minor AF (exact). Each radiograph was used to extract relevant features using K-means, Otsu method and Wavelet protocol. Thirtysix extracted features were used for training and the rest were used for evaluating the multi-layer Perceptron ANN model.

Results Analysis of the images from radiographs (test samples) by ANN showed that in 93\% of the samples, the location of the AF had been determined correctly by false rejection and acceptation error methods.

Conclusion Artificial neural networks can act as a second opinion to locate the $\mathrm{AF}$ on radiographs to enhance the accuracy of working length determination by radiography. In addition, ANN can function as a decision-making system in various similar clinical situations.

Keywords: apical foramen, artificial neural network, root canal treatment, working length.

Received 18 July 2011; accepted 21 September 2011

\section{Introduction}

Working length (WL) is the distance from a coronal reference point to the point at which canal preparation and filling should terminate. Correct WL determination and confining root filling materials only to the canal and not invading the periapical tissues

Correspondence: Mohammad Ali Saghiri, Department of Dental Material, Dental Branch, Islamic Azad University, Tehran, Iran (e-mail: saghiri@gmail.com). results in better root canal treatment outcomes and reduces the odds of insufficient cleaning of the full extent of the canal (Hedrick et al. 1994, Schaeffer et al. 2005). Instrumentation beyond the apical foramen (AF) should be avoided because it decreases the success rate (Sjogren et al. 1990, Smith et al. 1993). In the short term, accurate WL determination may prevent flare-ups (Georgopoulou et al. 1986), and in the long term it allows for successful treatment outcome by preventing periapical foreign body reactions (Nair et al. 1990), sealing of root canal 
apices (Wu et al. 2000a) and microbial control (Chugal et al. 2003).

The apical constriction (AC) forms the minor foramen (or minor diameter) and the most apical opening of the root canal is designated as the $\mathrm{AF}$ or major foramen or greater diameter. The distance between AC and AF varies from 0.5 to $0.7 \mathrm{~mm}$ (Ricucci \& Langeland 1998, Wu et al. 2000b). Biomechanical preparation should end at the AC (Ricucci \& Langeland 1998), where the contact between the root canal filling material and the apical tissue is minimal. The best prognosis for the root canal treatment is ensured when instrumentation terminates at the AC (Ricucci \& Langeland 1998, Wu et al. 2000b, Baugh \& Wallace 2005). Instrumentation beyond the AC (Swartz et al. 1983) or short of it (Seltzer et al. 1973) should be avoided because it reduces the success rate.

The radiographic apex is defined as the anatomical end of the root as seen on radiographs (Seltzer et al. 1973), whilst the AF is the region where the canal leaves the root surface and the AC often coincides with the cemento-dentinal junction. The AF deviates from the anatomic or radiographic apex in $60-94 \%$ of the cases (Kuttler 1955, Burch \& Hulen 1972). It is present 0.5-2.0 mm apical to the anatomic apex on the buccal, lingual or proximal surfaces. Studies show the distance between the AC and the anatomic apex on average $1 \mathrm{~mm}$ (Kuttler 1955, Dummer et al. 1984, McDonald \& Hovland 1990).

In clinical practice the AC cannot be detected radiographically. Moreover, a single distinct constriction that can be determined in a tactile approach is only present in 40-50\% of cases (Dummer et al. 1984). Although Olson et al. (1991) have reported that the location of the AF could be accurately determined radiographically, the distance from the AC cannot be measured. Despite the limited information provided by an intraoral radiograph, radiography remains an accepted and commonly used method for WL determination (Powell-Cullingford \& Pitt Ford 1993).

Electronic apex locators (EAL) are useful adjuncts in locating the terminus of the canal during root canal treatment. However, they cannot replace radiographic techniques. The ability of EAL to accurately locate the apex is 55-93\% (Seidberg et al. 1975, Busch et al. 1976, Trope et al. 1985, Fouad et al. 1990, McDonald \& Hovland 1990). Studies also indicate that false readings are often obtained from EAL, indicating the need for radiographic films (Seidberg et al. 1975, Busch et al. 1976).
Digital radiography has the advantages of lower radiation exposure to the patient, instant imaging without developing solutions or lengthy processing, ability to manipulate the digitized image for better observation (Hedrick et al. 1994, Almenar García et al. 1997, Burger et al. 1999), ease of radiographic data storage and, particularly in endodontics, instant display of the image (Heo et al. 2008). Real-time generation of high-resolution digital images, manipulation or processing of the captured image for enhanced diagnostic performance and digital documentation of patient records are some of the other advantages of digital radiography techniques (Naoum et al. 2003). The quality of the image is probably of utmost significance in endodontics because it facilitates accurate interpretation of root and canal morphology; in addition, it is important in the determination of radiographic canal length and the post-operative and long-term evaluation of the treatment outcome (Versteeg et al. 1997).

An artificial neural network (ANN) is a mathematical model inspired by the structure and/or functional aspects of biological neural networks in the brain. ANN is a decision-making system and helps the diagnostic procedure used for prediction of different elements from radiographs. ANNs are computer models with a massive parallel structure, which imitate the human brain (Lippmann 1987, McCulloch \& Pitts 1990). They consist of nonlinear computing elements, which represent neurons organized in several highly interconnected layers inspired by biologic neural networks. The basic idea is that even though artificial neurons have no intelligence individually, when interconnected, they might be able to duplicate aspects of the human brain combined with the computational power of computers. ANNs have proved extremely helpful in solving problems with high computational complexity, especially in the field of pattern recognition. Therefore, ANNs have been established as a promising alternative to statistical discriminate analysis because they can synthesize a considerable amount of information (or variables) without requiring statistical modelling of the problem. As medical diagnosis is a typical pattern recognition problem, previous applications have inspired the use of ANNs in the medical field such as diagnosis of breast cancer and coronary artery disease (Asada et al. 1990, Boone et al. 1990, Wu et al. 1992a,b, Tourassi \& Floyd 1993, Wu et al. 1993).

Artificial neural network is capable of 'learning' important relationships from a set of data and applying this knowledge to evaluate new cases. Instead of applying multiple rules or a fixed algorithm 
for differential diagnosis, ANN is particularly adept at classification problems, such as separating benign from malignant breast lesions because ANN is relatively insensitive to minor variations and noise within data (Boone et al. 1990, Wu et al. 1993). The structure of an ANN is a set of processing units (nodes) arranged in rows. Input nodes are interconnected by simple calculations with an internal layer of hidden nodes and a single output node. Rather than having a fixed algorithmic approach to a classification problem, an ANN is sequentially presented with a set of supervised training case-input data paired with the correct output. The ANN modifies its behaviour ('trains') by adjusting the strength or 'weights' of the connections until its own output converges to the known correct output. The information 'learned' by the ANN is stored in the weights of the network and is sent to connections between the nodes. Once trained, the network can evaluate a new case of input values by applying the weights learned from the data set with which it was trained. New cases do not have to be identical to the 'learned' cases; the network can generalize from the previous cases to evaluate cases not previously seen.

This study presents a new approach for locating the AF by using ANN to enhance the accuracy of working length determination with radiographs.

\section{Materials and methods}

Fifty single-rooted mandibular incisors and second premolars with curvatures $<30^{\circ}$ in the apical region, extracted for orthodontic or periodontal reasons, were collected and stored in $0.12 \%$ thymol solution. The teeth were placed in the empty tooth sockets of a dried skull in such a way that they fitted in the sockets as well as possible. Access cavities were prepared and working lengths were determined by placing a K-file (Dentsply Maillefer, Ballaigues, Switzerland) in the root canal and exposing a radiograph referred to as the 'in situ' radiograph by using a Rinn XCP (Dentsply Rinn, Elgin, IL, USA) and a photostimulable phosphorus system (Digora, Soredex, Tuusula, Finland). Then, the teeth were removed from the sockets. With the file stabilized in the access cavities using sticky wax, another radiograph was exposed, which was referred to as 'subtracted' radiograph for the learning procedure of the ANN system. Two endodontists evaluated the position of the files according to the position of the AF on the radiographs and then visualized the root apices under a stereomicroscope (Nikon Corporation, Tokyo,
Japan) equipped with a CCD camera at $\times 20$ and classified them into the following groups:

1. File tip was not visible under the stereomicroscope ('short').

2. The file tip was visible under the stereomicroscope but it was not visible more than $0.3 \mathrm{~mm}$ beyond the minor foramen ('exact').

3. The file was visible under the stereomicroscope and was beyond the minor foremen more than $0.3 \mathrm{~mm}$ ('long').

If they had different opinions about the position of the file, another endodontist evaluated the radiographs. At least two similar opinions were considered as the final decision. The features long, short or exact were used only for training the ANN System.

All the 'in situ' radiographs were matched by Picture Manager (Microsoft, Redmond, WA, USA) to $800 \times 600$ pixels. The images of the teeth on the radiographs were subtracted from the radiographs with the 'Otsu Method'. In this method, MATLAB (Mathworks Inc., Natick, MA, USA) software is used to import the images and their histograms are drawn with 256 characters (0-255). At the end of this phase, the outlines of the teeth were removed from the surrounding tissue images (Fig. 1a).

\section{Otsu method}

The Otsu method was used to automatically perform shape-based image thresholding histograms. This method can separate the teeth from the surrounding tissues based on differences in grey scales on radiographs. In the Otsu method there is a histogram plot for each picture. The teeth and surrounding tissues have many distinct features such as colour and intensity. The algorithm assumes that the image to be thresholded contains two classes of pixels. The white colour of the teeth produces one pinnacle in the histogram. In the Otsu method, grey scales lower than the peaks (threshold) are removed by first determining a threshold independently. Only differences between the white colour of the teeth and surrounding tissues remain. The Otsu method searches for a threshold that minimizes the intra-class variance, defined as a weighed sum of variances of the two classes (Gonzalez \& Woods 2002).

By finding the threshold, the algorithm can separate this threshold from other pixels on the image. In cases in which the amounts of black and white pixels were not in an appropriate scale and the Otsu method could not extract the teeth from surrounding tissues, 
(a)

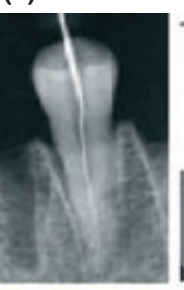

(f) $x=$ (b)
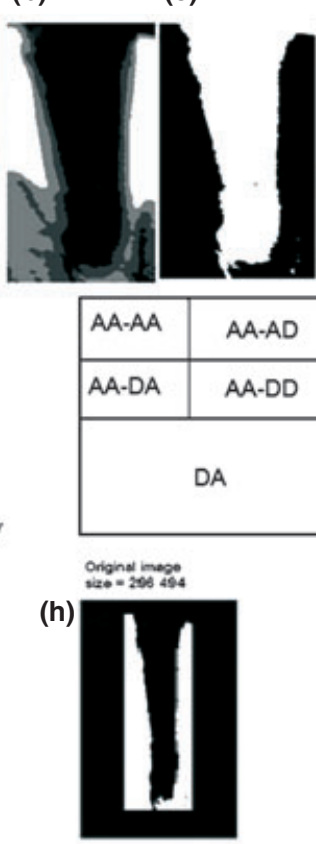

(k)

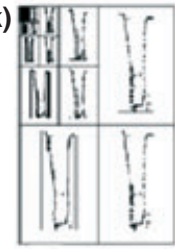

asgreal decombontion (d)

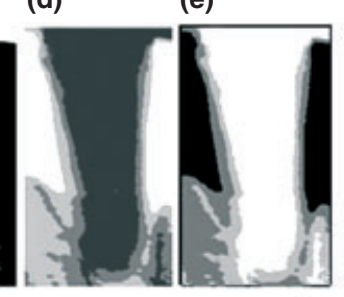

\begin{tabular}{|l|l|}
\hline DD-AA & DD-AD \\
\hline DD-DA & DD-DD \\
\hline
\end{tabular}

(g)

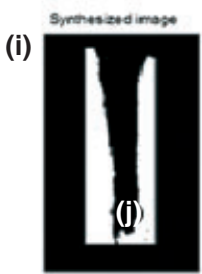

(I)

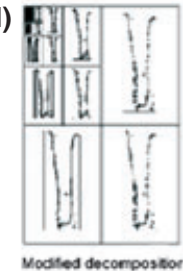

Nodited decompostor

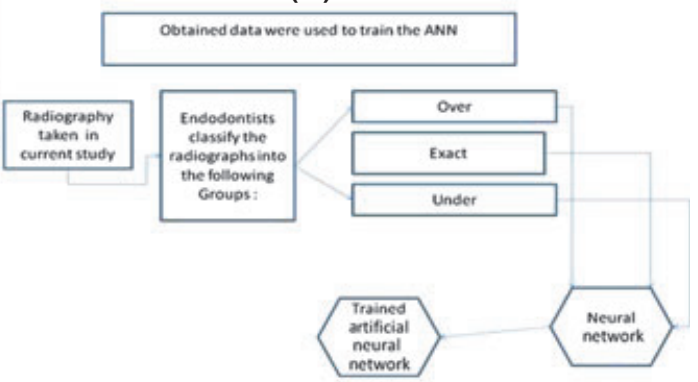

(m)

(n)
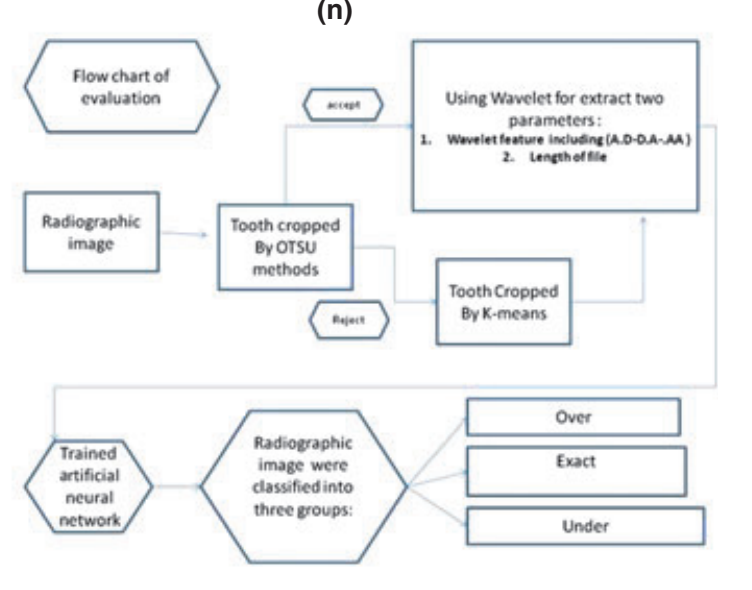

.

Figure 1 (a) The radiograph shows the tooth and the surrounding tissues; tooth images extracted from surrounding tissues with K-means classification. (b and d) Four grades of K-means cluster. (c and e) Combination of two classes with a high level of grey scale. White colour was used to show this class, and two other grey scales with black colour were combined to extract teeth from surrounding tissues. (f) Determining the length of teeth by counting the pixels. Counting started from point X towards point Y. The middle pixel was found and counting continued from the mid-pixel towards the most apical pixel $(\mathrm{Z})$. $(\mathrm{g})$ Schematic representation of 2D Wavelet from image D, detail; A, approximate ( $\mathrm{g}, \mathrm{h}$ ) Original image and coefficients of two-dimensional Wavelet form this image. (k) Original image coefficient of wave with two stages: (i) synthesized image from decomposition coefficient; (l) modified decomposition with Coiflets method; $(\mathrm{m}-\mathrm{n})$ flowcharts of feature extracting from radiographs and processing by artificial neural network.

K-means were used. Combinations of both MATLAB and K-means can crop the teeth from the surrounding tissues on the basis of means of variances (Gonzalez \& Woods 2002).

K-means clustering separates the teeth and surrounding tissues by differences in their colour. In the first step an algorithm is given. In an initial set of K-means $\mathrm{m}_{1}{ }^{(1)}, \ldots, \mathrm{m}_{\mathrm{k}}{ }^{(1)}$, the algorithm proceeds by alternating between two steps:

Assignment step: Assign each observation to the cluster with the closest distance.
Update step: Calculate the new means to be the centroid of the observations in the cluster, and update the centroid of each class (Hartigan 1975).

K-means classification was used with four classes with four centres; teeth, borders, surrounding tissues and empty areas around the teeth. The teeth and their borders were combined and categorized as white. In addition, areas around the teeth and surrounding tissues were categorized as black. The images were classified into four grey scale classes; black, white, dark grey and grey by K-means. Therefore, the areas around 
the teeth could be eliminated. Some white islands found in tooth images could be removed by morphological filters (Fig. 1b-e).

Morphological filters can remove the white islands (Gonzalez \& Woods 2002). First, the opening filter eliminates the islands. Then, closing filters can be used to eliminate black spots inside tooth images. Thus, tooth shapes are completely separated from other parts of the image. Finally, tooth shapes can be obtained as one of the features.

In the next stage, the 'in situ' radiographs were used for identifying other features. Tooth lengths were determined using 'counting' in MATLAB. To obtain high-quality images, referred to as 'detailed' and lowquality ones referred to as 'approximate', the 'Wavelet' in MATLAB was used. There were three features at the end of this stage: tooth length, ad approximate and detailed images. These features were submitted to multi-layer Perceptron ANN.

\section{Feature extraction}

\section{Tooth lengths}

In each specimen, an attempt was made to count from the middle pixel of teeth in the most coronal part towards the most apical pixel. In this way, tooth lengths could be obtained (Fig. 1f).

\section{Two-dimensional wavelet feature}

Two-dimensional Wavelet, sufficient for image processing, was used to extract the shape of the image as a feature (Antonini et al. 1992). Wavelet divided each image into four parts. Figure $1 \mathrm{~g}$ shows a schematic representation of two Wavelet functions with two decomposition processes. In this type of Wavelet, AA shows approximation in two dimensions whilst DD shows details of the image. AA has sufficient information about tooth shapes, and DD keeps sufficient information about image details. Wavelet transforms were used in detail and approximation again (two decomposition stages in detail and approximation). These features have the reduced sizes and approximate shapes of the images. Furthermore, details of images can be extracted as another feature. Original image, decomposition of the image and reconstructed image from coefficients can be seen in Fig. 1h-l. For each image, these extracted features were combined in a specific matrix of classification with the ANN. The processing and training flowcharts of the ANN can be seen in Fig. 1m-n.

\section{Multi-layer perceptron (MLP)}

Each of these features was classified as '001', '010' and '100'. From 50 'in situ' radiographs compared to the subtracted images imaged, 24 were categorized as 'long', 10 were categorized as 'exact' and the remaining as 'short'. Thirty-six of the specimens were used for training the network. The remaining specimens were used for testing the ANN. In the present study, a threelayer network was used for classification. Figure $2 \mathrm{a}$ depicts the performance of the neural network in 500 epochs with 0.02 error.

Briefly, the process to evaluate the reliability of the working length was as follows:

1. A digital radiograph was taken of the tooth, with an initial file placed at the estimated working length. The estimated working length was then rated visually.
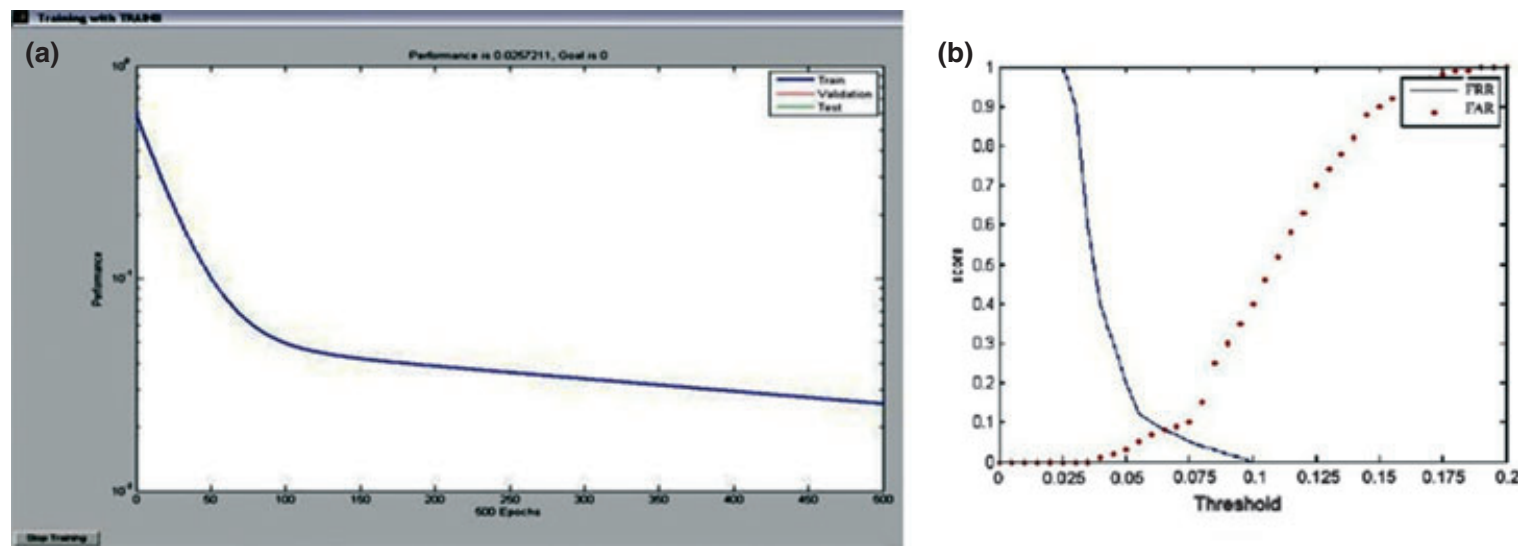

Figure 2 (a) Training plot for 500 epoch of neural network; Error of training: 0.02. (b) False rejection rate and false acceptance rate in different values of the threshold. 
2. The radiographic image was then processed using the Otsu method and K-means (described briefly in the 'Materials and methods') to yield a high contrast image of the tooth, with surrounding structures deleted. This image processing removed any image of the working length file and gave an outline only of the tooth itself. 3. The tooth length was then determined in MATLAB by counting pixels.

4. Tooth length and approximate and detailed images were then fed into 'Perceptron' and the reliability of the working length measurement was then decided by this system.

\section{Results}

Table 1 shows the results of testing process after training the ANN. There was only one error amongst 50 images, which was false-rejected from the 'short' category and false-accepted in the 'long' category. Figure $2 \mathrm{~b}$ shows the false rejection and false acceptance scores for different values of the thresholds.

Randomized distribution was used. For example, for training the network to recognize the file was short of the minor AF, 12 teeth were used and four teeth were used to test the ANN. For training in 'exact' classification, six teeth were used for training and four to test the ANN; for recognizing a file protruding beyond the minor AF (long), 18 teeth were used and six to test the ANN.

\section{Discussion}

Radiographs are indispensable in working length determination (Williams et al. 2006). The AF cannot be seen on radiographs; the only measuring point that can be viewed on radiographs is the anatomic apex (radiographic apex). Determination of the AC has traditionally been made by digital/tactile sensation and radiography (Seidberg et al. 1975). In young permanent teeth with immature apices, the apical dimension of the root canal is wider than that of the

Table 1 Number of teeth in each category of testing

\begin{tabular}{llrrrr}
\hline & $\begin{array}{c}\text { Number of } \\
\text { teeth in each } \\
\text { category }\end{array}$ & Training & Test & $\begin{array}{c}\text { False } \\
\text { rejection }\end{array}$ & $\begin{array}{c}\text { False } \\
\text { acceptation }\end{array}$ \\
\hline Short & 16 & 12 & 4 & 1 & 0 \\
Tip to tip & 10 & 6 & 4 & 0 & 0 \\
Long & 24 & 18 & 6 & 0 & 1 \\
Total & 50 & 36 & 14 & 1 & 1 \\
\hline
\end{tabular}

coronal two-thirds. In these cases, the endodontic file does not contact the apical dentinal walls of the root canal, and the EAL reading will usually be inaccurate (Hulsmann \& Pieper 1989). Although several studies have demonstrated that EALs can accurately determine the working length in 75.0-96.5\% of the root canals with mature apices (Fouad et al. 1990, McDonald \& Hovland 1990, Shabahang et al. 1996, Dunlap et al. 1998), only a few reports have described the use of an EAL in teeth with apical root resorption (Forsberg 1987, Surmont et al. 1992). Several studies have concluded that the use of a combination of methods to determine an appropriate working length may be more successful than relying on just one method (Dummer et al. 1984).

Although previous studies have shown no significant differences in identifying the file tip in extracted teeth compared with those integrated in a dried jaw (Olson et al. 1991), a dried skull was used in the present study to simulate clinical conditions. Likewise, no differences in the results were detected in the presence of soft tissues (gingiva and mucosa) (LeQuire et al. 1977). The ability to use computer-based systems to delete surrounding structures and isolate the tooth itself was simpler in the present study. Considering the clinical situation by testing real radiographs of teeth with several structures superimposed on them, it will be more difficult to generate similar images of maxillary molars, for example. Further studies must focus on resolving these shortcomings.

Examination of radiographs of 305 root canals on a viewing box showed that the AF could be accurately located in $82 \%$ of canals using only one radiograph per tooth; there was no significant difference in the alveolus of dried jaw specimens ( $86 \%$ flush) and extracted teeth $(78 \%$ flush) when radiographs were evaluated under a stereomicroscope (Olson et al. 1991).

A parallel radiograph is considered accurate for determining the location of the AF in human teeth during root canal instrumentation (Olson et al. 1991). A paralleling technique seems to provide a better reproduction of the distance between the apex and a contrasting subject (for example, a root canal instrument) than the bisecting-angle technique (Forsberg 1987). However, some studies have shown that the accurate determination or even estimation of the apical canal constriction is not possible with radiography because of anatomical variations or errors in projection (Surmont et al. 1992, ElAyouti et al. 2002). An initial file was used in the present study to determine the 
location of the $\mathrm{AF}$ in the training phase. In the test phase the presence of the initial file contributed to locating the AF.

Discrepancy between actual length and radiographic length will increase significantly as the degree of curvature increases (Kim-Park et al. 2003). In the present study, teeth with curvatures $<30^{\circ}$ in the apical third were selected. Such factors challenge the practitioner's clinical judgment in utilizing the two-dimensional radiograph in conjunction with the apex locator to determine the most ideal location as the end-point of instrumentation and canal filling. In addition, owing to shortage of input data for training, any intervention parameters must be minimized to prevent influencing the output value.

The teeth were classified at $\times 20$ magnifications along with $\pm 0.3 \mathrm{~mm}$. This classification was used for the 'training' phase of the ANN and owing to the learning pattern of the ANN it was not possible to classify the measurements in $\mathrm{mm}$ at this stage. This system functions as trained initially however, if the pattern of training is altered, it may be possible to measure these categories in mm. Nonetheless, a larger pool of data is needed, which might comprise at least one thousand separate information pieces, including periapical radiographs, stereomicroscopic images and opinion of endodontists for training of the ANN.

The Otsu method has an acceptable speed and accuracy in separating and cropping different objects on an image (Gonzalez \& Woods 2002). It cannot always extract teeth from the surrounding tissues owing to their close density, colour and grey scales. K-means clustering is a method of cluster analysis which tries to partition $\mathrm{n}$ observations (image grey scales) into $\mathrm{k}$ clusters. Each observation belongs to a cluster with the nearest mean. This method separates the teeth and surrounding tissues by colour differences. K-means method was used owing to its greater accuracy (Gonzalez \& Woods 2002).

The Wavelet method has many advantages in finding detail and approximation of images (Addison 2002). Using Wavelet transform has two advantages: Reduces the image size and extract properties and details from the tooth image (curvature and tooth border) (Antoine et al. 1993). Wavelet method was used in the present study to extract the detail and approximation of images.

Error of testing the system depends on the chosen threshold. The number of false rejections and false acceptances relies on choosing the threshold in accepting or rejecting the picture in one category. Intersec- tion between two curves gives the best threshold for rejection in one category (Jin et al. 2004).

This is a preliminary study, which assessed the suitability and feasibility of using an ANN to enhance decision-making in locating the AF. There appear to be two primary reasons why neural networks have not been applied in dentistry in the past. The first reason relates to the training requirements of the neural network. As the ability of the ANN to identify indications of an intrusion is completely dependent on the accurate training of the system, the training data and the training methods used are critical. Second, the training routine requires very large amounts of data to ensure that the results are statistically accurate.

In addition, the major advantage of ANNs is their ability to be used as an arbitrary function approximation mechanism that 'learns' from the observed data. This utility is particularly useful in applications where the complexity of the data or task makes impractical the design of such a function by human. It should be pointed out that ANN systems are closely dependent on the pool of data which is used for training; therefore, if the pool of data for training is increased, logically it should be more accurate (Asada et al. 1990).

The preliminary results from the experimental feedforward neural network give a positive indication of the potential offered by this approach, but a significant amount of research remains ahead before it can function as an effective detection system in endodontics.

\section{Conclusions}

A simple three-layer neural network can be trained to reliably identify the position of files in root canals with a noninvasive method. The subtracted pictures can help dentists categorize the location of the file tip in the root canal with minimum error. The subtracted data from additional radiographs are the best teacher for ANN in the training stage. The ANN diagnosis method can contribute to improving future diagnosis and leads to better outcomes in working length determination by radiography. In addition, ANN can act as a decisionmaking system in various similar clinical situations.

\section{Acknowledgements}

This publication was based on the post-PhD fellowship thesis by the first author to the Faculty of Endodontic Materials and Devices at Kamal Asgar Research Center 
(KARC) [center for excellence in endodontic material]. We are indebted to Professor Stephen C. Bayne for the provision of laboratory facilities in the Department of Dental and Biological Materials at the University of Michigan. Also special thanks to Drs. Hajar Afsar Ladjvardi, Sahar Dadvand and Majid Abdolrahimi for all of their contributions to this research. In addition, the authors thank Mr. Amir Pasha Mahmud Zadeh and Professor Ahmad Sheibani Nia for providing teeth and contributions. Special thanks are due to late professor Caro Lucas from School of Electrical \& Computer Engineering for his invaluable help and re-check of our network.

\section{Conflict of interest}

The authors deny any conflicts of interest related to this study.

\section{References}

Addison PS (2002) The illustrated wavelet transform handbook. Bristol: The Institute of Physics Publishing.

Almenar García A, Forner Navarro L, Ubet Castelló V, Miñana Laliga R (1997) Evaluation of a digital radiography to estimate working length. Journal of Endodontics 23, 363-5.

Antoine JP, Carrette P, Murenzi R, Piette B (1993) Image analysis with two-dimensional wavelet transform. Signal Processing 31, 241-72.

Antonini M, Barlaud M, Mathieu P, Daubechies I (1992) Image coding using wavelet transform. IEEE Transactions on Image Processing 1, 205-20.

Asada N, Doi K, MacMahon $\mathrm{H}$ et al. (1990) Potential usefulness of an artificial neural network for differential diagnosis of interstitial lung diseases: pilot study. Radiology 177, 857-60.

Baugh D, Wallace J (2005) The role of apical instrumentation in root canal treatment: a review of the literature. Journal of Endodontics 31, 333-40.

Boone JM, Sigillito VG, Shaber GS (1990) Neural networks in radiology: an introduction and evaluation in a signal detection task. Medical Physics 17, 234-41.

Burch JG, Hulen S (1972) The relationship of the apical foramen to the anatomic apex of the tooth root. Oral Surgery Oral Medicine Oral Pathology Oral Radiology and Endodontics 34, 262-8.

Burger CL, Mork TO, Hutter JW, Nicoll B (1999) Direct digital radiography versus conventional radiography for estimation of canal length in curved canals. Journal of Endodontics 25, 260-3.

Busch LR, Chiat LR, Goldstein LG, Held SA, Rosenberg PA (1976) Determination of the accuracy of the Sono-Explorer for establishing endodontic measurement control. Journal of Endodontics 2, 295-7.

Chugal NM, Clive JM, Spångberg LS (2003) Endodontic infection: some biologic and treatment factors associated with outcome. Oral Surgery Oral Medicine Oral Pathology Oral Radiology and Endodontics 96, 81-90.

Dummer PM, McGinn JH, Rees DG (1984) The position and topography of the apical canal constriction and apical foramen. International Endodontic Journal 17, 192-8.

Dunlap CA, Remeikis NA, BeGole EA, Rauschenberger CR (1998) An in vivo evaluation of an electronic apex locator that uses the ratio method in vital and necrotic canals. Journal of Endodontics 24, 48-50.

ElAyouti A, Weiger R, Löst C (2002) The ability of root ZX apex locator to reduce the frequency of overestimated radiographic working length. Journal of Endodontics 28 , 116-9.

Forsberg J (1987) Radiographic reproduction of endodontic "working length" comparing the paralleling and the bisecting-angle techniques. Oral Surgery Oral Medicine Oral Pathology Oral Radiology and Endodontics 64, 353-60.

Fouad AF, Krell KV, McKendry DJ, Koorbusch GF, Olson RA (1990) Clinical evaluation of five electronic root canal length measuring instruments. Journal of Endodontics 16, 446-9.

Georgopoulou M, Anastassiadis P, Sykaras S (1986) Pain after chemomechanical preparation. International Endodontic Journal 19, 309-14.

Gonzalez RC, Woods RE (2002) Digital image processing, 2nd edn. Englewood Cliffs, NJ: Prentice-Hall.

Hartigan JA (1975) Clustering algorithms. New York: John Wiley.

Hedrick RT, Dove SB, Peters DD, McDavid WD (1994) Radiographic determination of canal length direct digital radiography versus conventional radiography. Journal of Endodontics 20, 320-6.

Heo MS, Han DH, An BM et al. (2008) Effect of ambient light and bit depth of digital radiograph on observer performance in determination of endodontic file positioning. Oral Surgery Oral Medicine Oral Pathology Oral Radiology and Endodontics 105, 239-44.

Hulsmann M, Pieper K (1989) Use of an electronic apex locator in the treatment of teeth with incomplete root formation. Endodontics \& Dental Traumatology 5, 238-41.

Jin ATB, Ling DNC, Song OT (2004) An efficient fingerprint verification system using integrated wavelet and FourierMellin invariant transform. Image and Vision Computing 22, 503-13.

Kim-Park MA, Baughan LW, Hartwell GR (2003) Working length determination in palatal roots of maxillary molars. Journal of Endodontics 29, 58-61.

Kuttler Y (1955) Microscopic investigation of root apexes. Journal of the American Dental Association 50, 544-52.

LeQuire AK, Cunningham CJ, Pelleu GB Jr (1977) Radiographic interpretation of experimentally produced osseous 
lesions of the human mandible. Journal of Endodontics 3, 274-6.

Lippmann RP (1987) An introduction to computing with neural nets. IEEE ASSP Magezine 4, 4-22.

McCulloch WS, Pitts W (1990) A logical calculus of the ideas immanent in nervous activity. Bulletin of Mathematical Biology 52, 99-115.

McDonald NJ, Hovland EJ (1990) An evaluation of the apex locator endocater. Journal of Endodontics 16, 5-8.

Nair PN, Sjögren U, Krey G, Sundqvist G (1990) Therapyresistant foreign body giant cell granuloma at the periapex of a root-filled human tooth. Journal of Endodontics 16, 58995.

Naoum HJ, Chandler NP, Love RM (2003) Conventional versus storage phosphor-plate digital images to visualize the root canal system contrasted with a radiopaque medium. Journal of Endodontics 29, 349-52.

Olson AK, Goerig AC, Cavataio RE, Luciano J (1991) The ability of the radiograph to determine the location of the apical foramen. International Endodontic Journal 24, 28-35.

Powell-Cullingford AW, Pitt Ford TR (1993) The use of E-speed film for root canal length determination. International Endodontic Journal 26, 268-72.

Ricucci D, Langeland K (1998) Apical limit of root canal instrumentation and obturation, part 2. A histological study. International Endodontic Journal 31, 394-409.

Schaeffer MA, White RR, Walton RE (2005) Determining the optimal obturation length: a meta-analysis of literature. Journal of Endodontics 31, 271-4.

Seidberg BH, Alibrandi BV, Fine H, Logue B (1975) Clinical investigation of measuring working lengths of root canals with an electronic device and with digital-tactile sense. Journal of American Dental Association 90, 379-87.

Seltzer S, Soltanoff W, Smith J (1973) Biologic aspects of endodontics. V. Periapical tissue reactions to root canal instrumentation beyond the apex and root canal fillings short of and beyond the apex. Oral Surgery, Oral Medicine, and Oral Pathology 36, 725-37.

Shabahang S, Goon WW, Gluskin AH (1996) An in vivo evaluation of Root ZX electronic apex locator. Journal of Endodontics 22, 616-8.

Sjogren U, Hagglund B, Sundqvist G, Wing K (1990) Factors affecting the long-term results of endodontic treatment. Journal of Endodontics 16, 498-504.
Smith CS, Setchell DJ, Harty FJ (1993) Factors influencing the success of conventional root canal therapy - a five-year retrospective study. International Endodontic Journal 26, 321-33.

Surmont P, D'Hauwers R, Martens L (1992) Determination of tooth length in endodontics. Revue Belge de Medecine Dentaire 47, 30-8.

Swartz DB, Skidmore AE, Grieffin JA (1983) Twenty years of endodontic success and failure. Journal of Endodontics 9, 198-202.

Tourassi GD, Floyd CE Jr (1993) Artificial neural networks for single photon emission computed tomography. A study of cold lesion detection and localization. Investigative Radiology 28, 671-7.

Trope M, Rabie G, Tronstad L (1985) Accuracy of an electronic apex locator under controlled clinical conditions. Endodontics \& Dental Traumatology 1, 142-5.

Versteeg KH, Sanderink GC, van Ginkel FC, van der Stelt PF (1997) Estimating distances on direct digital images and conventional radiographs. Journal of American Dental Association 128, 439-43.

Williams CB, Joyce AP, Roberts S (2006) A comparison between in vivo radiographic working length determination and measurement after extraction. Journal of Endodontics $\mathbf{3 2}$, $624-7$.

Wu CY, Giger ML, Doi K et al. (1992a) Application of artificial neural networks in mammography for the diagnosis of breast cancer. Proceedings of SPIE 1778, 19-27.

Wu Y, Doi K, Giger M, Nishikawa RM (1992b) Computerized detection of clustered microcalcifications in digital mammograms: applications of artificial neural networks. Medical Physics 19, 555-60.

Wu Y, Giger ML, Doi K, Vyborny CJ, Schmidt RA, Metz CE (1993) Artificial neural networks in mammography: application to decision making in the diagnosis of breast cancer. Radiology 187, 81-7.

Wu MK, Fan B, Wesselink PR (2000a) Leakage along apical root fillings in curved root canals. Part I: effects of apical transportation on seal of root fillings. Journal of Endodontics 26, 210-6.

Wu MK, Wesselink PR, Walton RE (2000b) Apical terminus location of root canal treatment procedures. Oral Surgery Oral Medicine Oral Pathology Oral Radiology and Endodontics 89, 99-103. 